\title{
Floral structure for textile-based metamaterial absorber
}

\author{
Siti Nurzulaihan Isa, Osman Ayop, Farid Zubir, Noor Asniza Murad, Mohammad Kamal A. Rahim \\ Advanced RF and Microwave Research Group, Department of Communication Engineering, \\ Universiti Teknologi Malaysia, Malaysia
}

\begin{tabular}{l} 
Article Info \\
\hline Article historys: \\
Received Mar 27, 2019 \\
Revised May 6, 2019 \\
Accepted May 29, 2019 \\
\hline Keywords: \\
Absorber \\
Flexible \\
Metamaterial \\
Motion detector \\
Polarization insensitive
\end{tabular}

\begin{abstract}
Based on an annulled circle structure metamaterial absorber (MMAb), a floral structure MMAb design is proposed for performance improvement especially on absorptivity. Since the initial purpose of designing the MMAb is for hiding one's existence from electromagnetic motion detector, it is expected that the MMAb will has flexibility as one of its characteristics. The MMAb composes of textile-based substrate in between floral structure at the top and ground plane at the bottom, based on metallic plate. The targeted center frequency is $10.525 \mathrm{GHz}$ and through series of simulation done in CST Microwave Software, the MMAb can reach up to $97.03 \%$ of absorptivity. The operating angle can be extended at least $68 \mathrm{o}$ before its absorptivity deteriorated below $80 \%$. In addition, the proposed MMAb has a high absorptivity regardless of the polarization angle of the electromagnetic waves.
\end{abstract}

Copyright (c) 2019 Institute of Advanced Engineering and Science. All rights reserved.

\section{Corresponding Author:}

Osman Ayop,

Department of Communication Engineering,

School of Electrical Engineering,

Universiti Teknologi Malaysia,

81310 Johor Bahru, Malaysia.

Email: osman@fke.utm.my

\section{INTRODUCTION}

Electromagnetic absorber functions to absorb all incident radiations at the operating frequency. Some of the devices that can radiate electromagnetic radiation are radar and motion detector. This paper will focus on how a microwave absorber can 'sneak away' from being detected by motion detector. Based on recent market study, many of motion detectors operate at X-Band frequency, and one of them is at $10.525 \mathrm{GHz}$, functioning in the range of three to seven meters from the detector [1]. In other word, MMAb has lots of similarity to an invisibility cloak $[2,3]$. Some of other devices use of MMAb are bolometer spectroscopy $[4,5]$, thermal emission $[6,7]$, and sub-wavelength imaging $[8,9]$. However, working mechanism of MMAb requires all incident radiation to be absorbed at the operating frequency by using the concept of transmission line theory. In that theory, metallic plate functions as short circuit and the placement of other metallic layer with a certain distance relating to the operating wavelength will act as a load, therefore an open-circuit scenario is established [10]. As an absorber that absorbs all the electromagnetic radiations, a very minimal reflectance is required because the incident wave can only see the metallic patch as admittance. When the load impedance matches the free space, the reflectivity will be zero, therefore high absorptivity can be realized. To design a structure whose load impedance matches the free space is one of the challenges in designing a MMAb.

There were some researches that worked on MMAb structural design and some analysis have been made from the works [11-16]. However, most of them using FR-4, which is hard and not flexible, as substrate materials. Therefore, the degree of flexibility is very low especially for wearable application. To create a $\mathrm{MMAb}$ that function against a motion detector means that it needs flexibility to operate against all incident waves, including some bending effects on no matter polarization it might be. The most suitable material to operate on is textile material. However, since the design MMAb operates in X-Band, a unit cell is predicted to be very small, therefore a very light and thin metallic plate is needed to achieve the purpose. 
The advancement of MMAb study has widened the variation of the absorber itself to be, for example, dual-, multiband, tuneable and flexible. The study of flexible MMAb such as [17] by D. Lee et. al. although not operating at X-Band provides idea on how a very small and detailed MMAb can be realized, that is through 3-D printing. Another example of flexible MMAb is [18] by J. Tak et. al. operates at dual-band based on felt, which is textile-based. However the model has low absorptivity at oblique incident angle.

In this paper, a flexible textile metamaterial absorber is design based on floral-structure. The peak absorbance of the structure can achieve above $97 \%$. The operating angle is also large where it can maintain the absorbance more than $80 \%$ for incident angle as large as $68^{\circ}$.

\section{DESIGN CONSIDERATION}

\subsection{Selecting the material for flexibility purpose}

To achieve the purpose of stealthy against a motion detector, the MMAb is aimed to be light and flexible. The best material for the task is textile and a very thin metallic plate. The textile substrate is projected to be $1.0 \mathrm{~mm}$ thin and dielectric constant, $\mathrm{gr}$ of 1.7 with tangent loss of 0.02 , operating at $2.45 \mathrm{GHz}$. The closest material would be denim. The copper plate has an electric conductivity of $5.8 \times 10^{7} \mathrm{~S} / \mathrm{m}$ with the thickness of $0.035 \mathrm{~mm}$. The rational of choosing the thinnest material possible is based on the study that the greater absorptivity can be achieved through thinner absorber [10].

\subsection{Structural design of MMAbs}

Several structures were designed to find out the maximum possible absorptivity that can be achieved. This paper proposes the final design of the MMAb that is the result form increasing the complexity of the basic design of an annulled circle MMAb. Through the design process, the top structure is designed based on the performance of the absorptivity from the simulation. The final design of the MMAb is shown in Figure 1. The dimensions of the design is recorded on Table 1.The top view of the MMAb is presented in Figure 1, and as the visual suggests, the model is called floral MMAb. The parameter of the MMAb is explained in Table 1. Some parameters have fixed values because of the material used itself, for example the thickness of both metallic plate and textile-based substrate, as stated in previous sub-chapter.

Using the same mechanism as annulled circular structure, symmetrical factor of the structure is used for the basic design of the MMAb because of based on parametric reviews on [11, 12, 19], a symmetrical structure gives an advantage of being insensitive to polarization. On top of that, as this MMAb aims to be flexible, the symmetrical structure gives an advantage of being less affected to the change of incident angle. In the course of the simulation, the analysis of the MMAb is compared to the model of annulled circle MMAb, which has the same material and adjusted patch size to the the same resonant frequency [12].

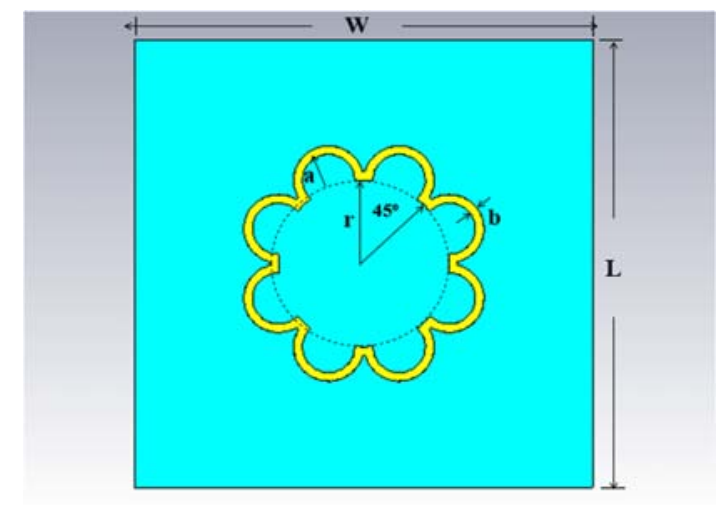

Figure 1. The design of the floral MMAb

Table 1. Reference parameter

\begin{tabular}{cc}
\hline Label & Width $(\mathrm{mm})$ \\
\hline$W$ & 13.3 \\
$L$ & 13.3 \\
$a$ & 1.02 \\
$b$ & 0.25 \\
$r$ & 2.45 \\
\hline
\end{tabular}

Indo. J. Elec. Eng. \& Inf, Vol.7, No. 2, June 2019: 375 - 381 


\section{PARAMETRIC STUDY}

The first sub-section provides the simulated reflectance, $R(\omega)$, transmittance, $T(\omega)$, and absorbance, $\mathrm{A}(\omega)$ in linear form. Then, a series of simulation at different incident angle was conducted to record the performance of the absorptivity. To test whether the MMAb is insensitive towards the change of polarization angle, the absorber is rotated up to $90 \mathrm{o}$ at the plane parallel to the port.

\subsection{Reflectance, transmittance and absorbance}

Figure 2 illustrates the three parameters when the phi and theta is 00 , which indicates the EM radiation at the electrical field. The figure shows that at the center frequency of $10.525 \mathrm{GHz}$, the absorbance, $\mathrm{A}(\omega)$ peaks up to $97.00 \%$, whereas the reflectance, $R(\omega)$ is at its minimum, which is $3.00 \%$. The transmittance, $T(\omega)$ with the percentage of $0.010 \%$ insignificantly affects the absorbance of the MMAb. As for the performance at the magnetic field, all the three parameters show similar behaviour, however differ in values. The reflectance, $R(\omega)$ reaches the lowest that is $2.97 \%$, while the absorbance, $\mathrm{A}(\omega)$ is at the maximum of $97.03 \%$. The transmittance, $\mathrm{T}(\omega)$ is $0.011 \%$, which is within the range of expected values (near zero). Both simulations show that the proposed MMAb exhibit the fundamental characteristic of a good absorber, which is, firstly the reflectance, $\mathrm{R}(\omega)$ and the absorbance, $\mathrm{A}(\omega)$ will always contradict with each other, secondly, transmittance, $\mathrm{T}(\omega)$ will always near zero.

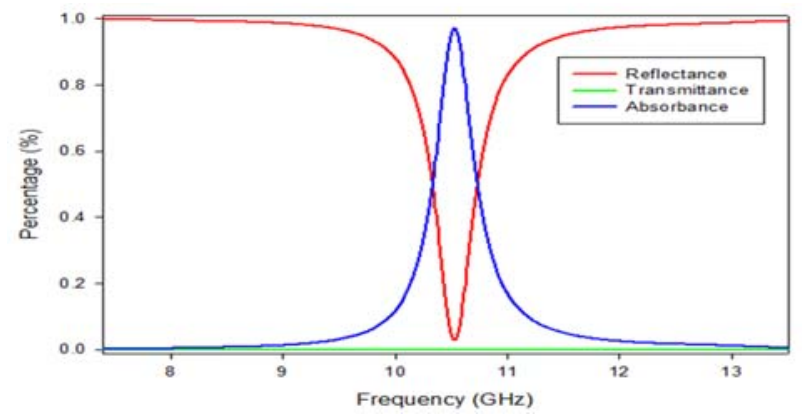

Figure 2. Reflectance, transmittance and absorbance at TE-Field

\subsection{Absorbance for different incident angle}

Figure 3 illustrates the simulated absorbance of the MMAb for TE polarization of different incident angles. Due to a very small margin of differences, the MMAb was simulated at only four incident angles to avoid confusion, that are at $0^{\circ}, 30^{\circ}, 50^{\circ}$, and $70^{\circ}$, For TE Polarization, the absorbance reaches maximum at the center frequency by $97.06 \%, 99.73 \%, 97.09 \%$ and $73.73 \%$ for $0^{\circ}, 30^{\circ}, 50^{\circ}$, and $70^{\circ}$ angle of incidents respectively. The peak of absorptivity is when the angle of incident is $30^{\circ}$.

As predicted from previous simulations, simulated absorbance of the MMAb for TM polarization of different incident angles show similar result with TE polarization. The absorbances recorded at $10.525 \mathrm{GHz}$ are $97.04 \%, 99.73 \%, 97.08 \%$ and $73.83 \%$ for $0^{\circ}, 30^{\circ}, 50^{\circ}$, and $70^{\circ}$ angle of incidents respectively. Absorbance for both TE and TM polarization achieve maximum magniture at the incident angle of $30^{\circ}$.

Further investigation the absorptivity over changing incident angle, the proposed MMAb shows a consistent behavior of high absorptivity, which is above $90 \%$ until $59^{\circ}$, before deteriorating. However it still maintains a relative good absorptivity of at least $80 \%$ at $66^{\circ}$.

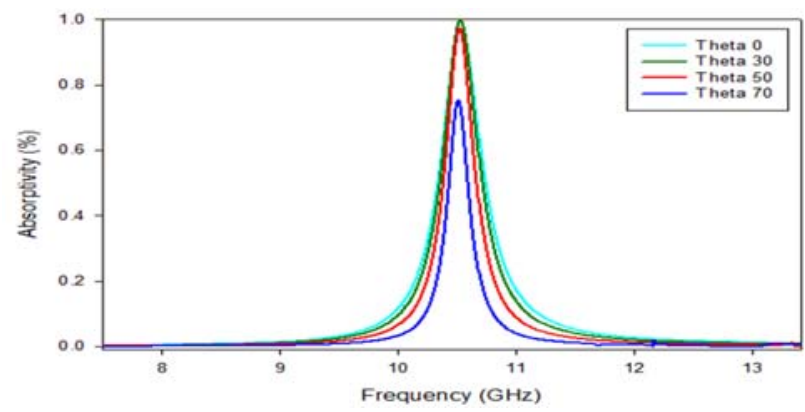

Figure 3. Absorptivity for different incident angle

Floral structure for textile-based metamaterial absorber (Siti Nurzulaihan Isa) 


\subsection{Absorbance for different polarization angle}

The absorptivity along the change of polarization angle is shown in Figure 4 . As the design is horizontally and vertically symmetrical at top view of the MMAb, the simulation only took place from $0^{\circ}$ to $90^{\circ}$. The absorptivity achieved by the proposed MMAb fluctuates very slightly, that although it has five samples of angle, which are $0^{\circ}, 22 .^{\circ}, 45^{\circ}, 67.5^{\circ}$ and $90^{\circ}$, it appears to be consistent in Figure 5, as the lines of each angle overlapped with each other. This figure insinuates that the absorptivity seems to be unaffected to polarization change. The divergence of the absorptivity is $0.9 \%$ throughout the polarization angle, compared to annulled circle model that has deviation of $1.84 \%$ in absorptivity [4]. From these two figures, it can be assured that both of the MMAb designs are insensitive towards polarization as the deviation is too little to put into consideration.

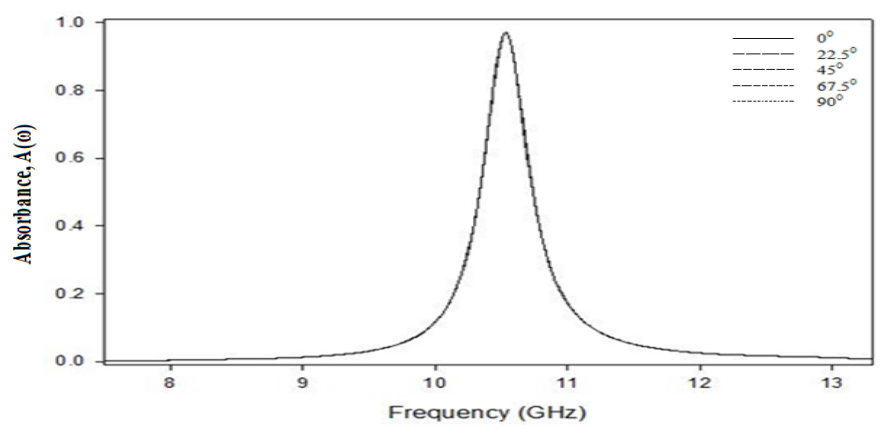

Figure 4. Absorptivity over changing polarization angle

\subsection{Simulated current distribution and surface current}

The field distribution and surface current are studied at $10.525 \mathrm{GHz}$ through field monitors function in the CST software. The simulated electrical field distribution is shown in Figure 5(a) while the simulated magnetic field distribution is shown in Figure 5(b).

At $10.525 \mathrm{GHz}$, the E-Filed distribution is very strong at vertical part of the MMAb. This is an indication of strong electric coupling exists between two parallel metal elements. The flow of the E-Field is getting weaker towards the horizontal axis of the MMAb. The high magnitude of the E-Field distribution is concentrated at the middle part of metallic plane, which is at the center of vertical axis of the MMAb. It is also found that the weaker current distribution gathers around the stronger induced field.

Referring to Figure 5(b), the field monitor shows a similar behavior with the previous E-Field distribution, however, instead of concentrating on vertical axis, the H-Field distribution intensifies on the horizontal axis and getting weaker towards vertical axis. Hence, it can be concluded that a stronger induced field achievable along the direction of its respective incident field, which are $\mathrm{Y}$ axis for E-Field and $\mathrm{X}$ axis for $\mathrm{H}$-Field. Every part of the circular ring cannot exhibit the same magnitude for both E- and H-fields as they are placed perpendicular with each other by nature.

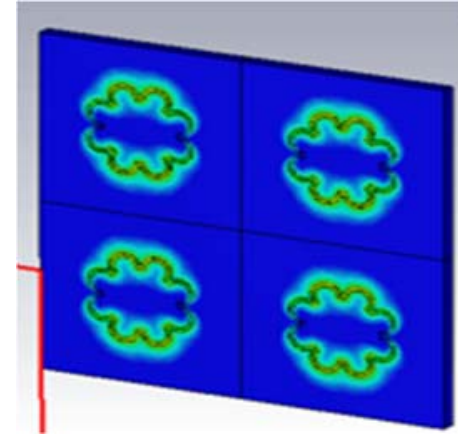

(a)

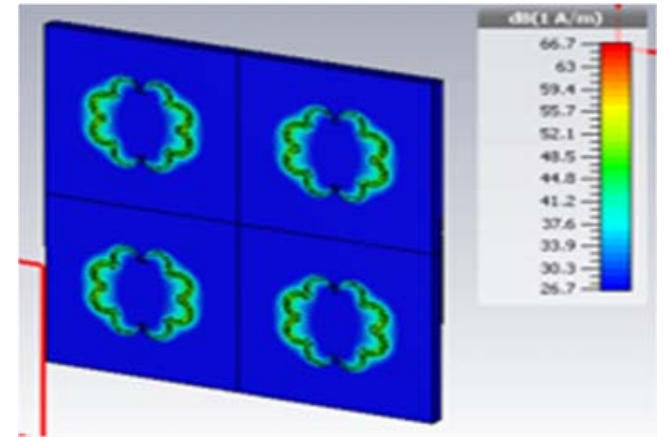

(b)

Figure 5. Field distribution for (a) E-field and (b) H-field 
Figure 6 supposedly illustrates the flow of the current on the bottom surface of MMAb. It is shown that the flows move up and down in phase. The higher energy concentrated at the horizontal axis of the MMAb shows that the surface currents are related to H-Field, therefore, this proves the stronger current at the axis of its respective field. The actual simulation is that, the surface current flow is separately simulated for resonating elements and combined element. It is found out that direction of the currents flow is always anti-parallel at $10.525 \mathrm{GHz}$. Comparing to the annulled circle model, the energy of the floral MMAb is relatively higher [4]. It is assumed that the more complex structure contributes to the higher energy flow on the field distribution.

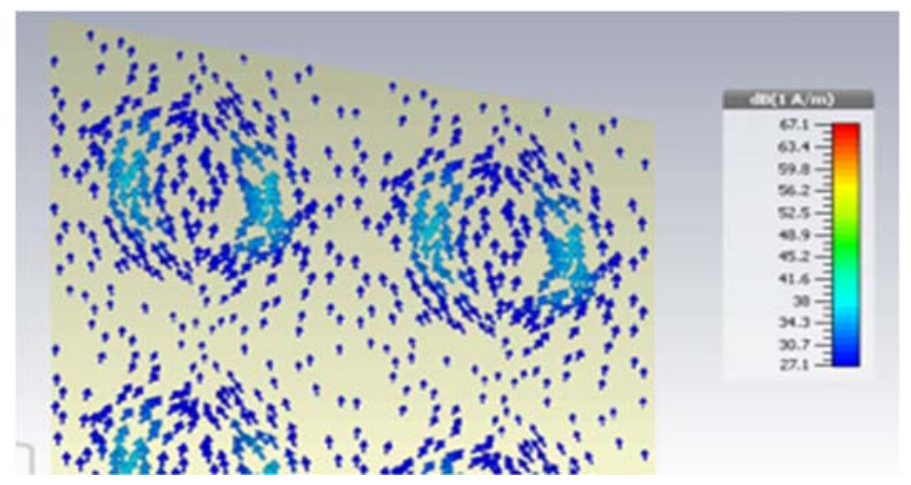

Figure 6. Surface current flow of the bottom structure of the MMAb

\subsection{The bending effect of the performance of floral MMAb}

The effect of bending the structure of Floral MMAbs are studied. Figure 7(a) shows the convex bending while Figure 7(b) shows a concave bending. The bending factor for both concave and convex is presented in Table 2. The results for these two types of bending are presented in Figure 8. From the results, it shows that the absorption are still high for all bending factors however, its have a slight variation of absorption frequency.

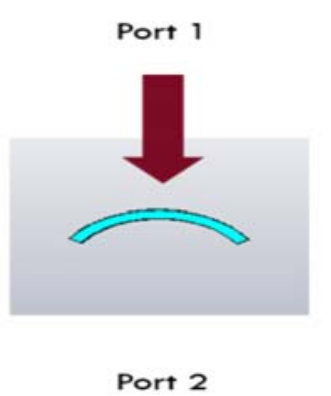

(a)

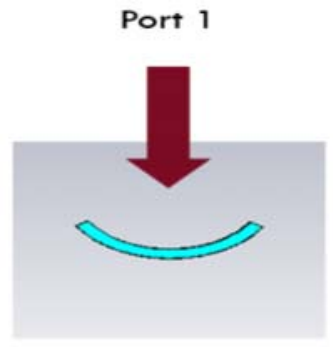

Port 2

(b)

Figure 7. (a) Convex bending and (b) concave bending

Table 2. Bending factor

\begin{tabular}{ccc}
\hline $\begin{array}{c}\text { Culvature } \\
\text { radius }\end{array}$ & $\begin{array}{c}\text { Bending Factor } \\
\text { (Concave) }\end{array}$ & $\begin{array}{c}\text { Bending Factor } \\
\text { (Convex) }\end{array}$ \\
\hline $5 / 3 * \mathrm{~W}$ & 1.7 & 1.7 \\
$3 / 2 * \mathrm{~W}$ & 1.5 & 1.5 \\
$5 / 4 * \mathrm{~W}$ & 1.3 & 1.3 \\
$\mathrm{~W}$ & 1.0 & 1.0 \\
$3 / 4 * \mathrm{~W}$ & 0.7 & 0.7 \\
$1 / 2 * \mathrm{~W}$ & 0.5 & 0.5 \\
$1 / 3 * \mathrm{~W}$ & 0.3 & 0.3 \\
\hline Note: $\mathrm{W}=$ the width of the unit cell of Floral MMAb.
\end{tabular}

Floral structure for textile-based metamaterial absorber (Siti Nurzulaihan Isa) 


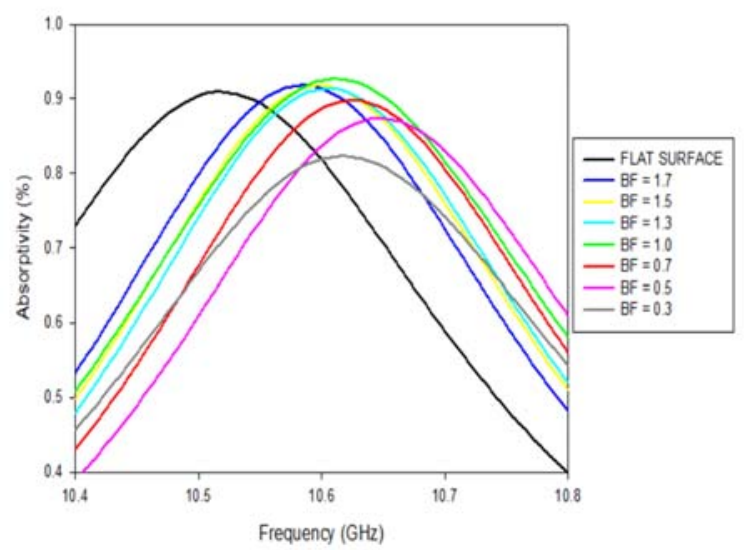

(a)

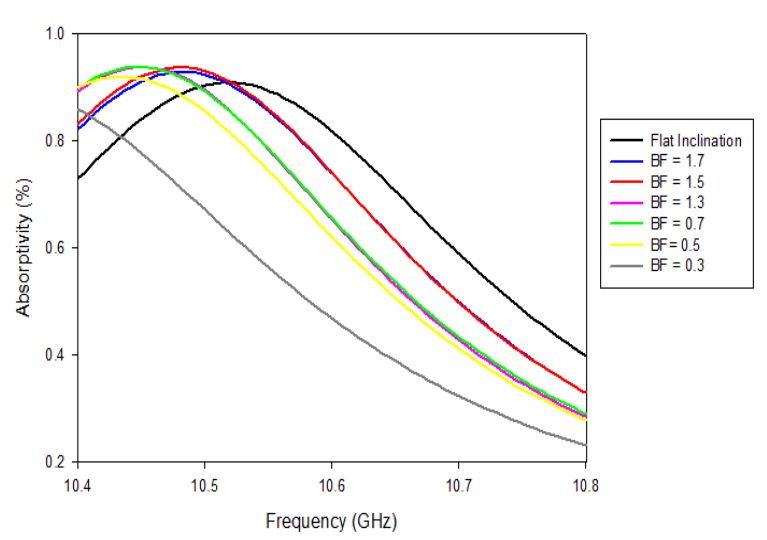

(b)

Figure 8. Absorbance for different bending factor: (a) Convex bending and (b) Concave bending

\section{CONCLUSION}

Parametric study has been conducted throughout the design process and the performance of the $\mathrm{MMAb}$ is observed through series of simulations. From the simulation, it can be concluded that proper element size should be used to obtain high absorptivity. The structure of the MMAb always contributes to the behavior of the resonant frequency. Therefore, the design has to serve the aimed functions of the MMAb. For example, a single line in relative to polarization will exhibit single-band MMAb's nature. Based on current distribution monitoring, loss of power at the surface of the MMab gets larger due to a more complex structure. Hence, weaken its ability to reflect resulting to an increase in absorptivity. That explains the better absorptivity of floral MMAb compared to the simple design. Finally, the bending study shows that the high absorptivity can still be achived with slight shift on resonant frequency.

\section{ACKNOWLEDGMENTS}

The authors thank the Ministry of Education (MOE) for supporting the research work and Research Management Centre (RMC), School of Postgraduate Studies (SPS) and School of Electrical Engineering (SKE), Faculty of Engineeering, Universiti Teknologi Malaysia (UTM), Johor Bahru, for the support of the research under Grant Nos. 14J87. The authors would also like to acknowledge all members of Advanced RF and Microwave Research Group (ARFMRG). 


\section{REFERENCES}

[1] J. C. Whitaker, The resource handbook of electronics. CRC Press LLC. 2001; A247: 31.

[2] Shengying Liu, Qun Wu, and Kuang Zhang, "Dispersion effect on electromagnetic properties of metamaterials invisibility cloak," Cross Strait Quad-Regional Radio Science and Wireless Technology Conference, pp. 226-229, 2011.

[3] Yu Luo, Jingjing Zhang, and Hongsheng Chen, "Analysis on a perfect cylindrical cloak realizable with two dimensional metamaterials," 2008 International Workshop on Metamaterials, pp. 129-132, 2008.

[4] Kamil Boratay Alici, "Hybridization of fano and vibrational resonances in surface-enhanced infrared absorption spectroscopy of streptavidin monolayers," IEEE Transaction on Nanotechnology, vol. 13, no. 2, pp. 216-221, 2014.

[5] S. A. Wolf, U. Strom, J. C. Culbertson, and D. Paget, "Superconducting granular NBN bolometer for ultrafast spectroscopy," 2012 IEEE Transaction on Magnetics, vol. 21, no. 2, pp. 920-923, 2012.

[6] Qinhuo Liu, Huaguo Huang, Wenhan Qin, Kaihua Fu, and Xiaowen Li, "An extended 3-D radiosity-graphics combined model for studying thermal-emission directionality of crop canopy," IEEE Transaction on Geoscience and Remote Sensing, vol. 45, no. 9, pp. 2900-2918, 2007.

[7] Jindi Wang, Xiaowen Li, Hongbo Su, and Ziti Jiao, "Approach on directional thermal emission modeling for 3-D structural pixel and the model validation," 2001 International Geoscience and Remote Sensing Symposium, vol. 4, pp. 1985-1987, 2001.

[8] Madhuja Ghosh and Subal Kar, "Metamaterial plane-slab focusing and sub-wavelength imaging - The concept, analysis and characterization," Science and Information Conference 2013, pp. 670-674. 2013.

[9] Yan Chen, Xiaohua Teng, and Yijun Feng, "Loss effect on sub-wavelength imaging by compensated bilayer of anisotropic metamaterials," IEEE Antennas and Propagation Society International Symposium 2006, pp. 3361-3364, 2006.

[10] C. A. Balanis, Antenna Theory: Analysis and design, 3ed. Wiley Interscience. 2005.

[11] D. Lim, D. Lee, and S. Lim, “Angle- and polarization-insensitive metamaterial absorber using via array,” Sci. Rep. 6, 39686; DOI: 10.1038/srep39686. 2016.

[12] O. Ayop, M. K. A. Rahim, and N. A. Murad, "Wide angle and polarization insensitive circular ring metamaterial absorber at $10 \mathrm{GHz}$, , Appl. Phys. A., vol. 122(374). 2016.

[13] N. I. Landy, S. Sajuyigbe, J. J. Mock, D. R. Smith, and W. J. Padilla, "A perfect metamaterial absorber," Phys. Rev. Lett., 100: 207402. 2008.

[14] J. Q. Feng, L. Si, L. Sun, Y. Tian, H. Huang, Y. T. Jin, et al., "An ultrathin polarization-independent wideband metamaterial absorber for EMC applications," EMC EUROPE, 2017 International Symposium, 2017.

[15] D. Lee, H. K. Sung, and S. Lim, "Flexible subterahertz metamaterial absorber fabrication using inkjet printing technology,” Appl. Phys. B., 2016, Springer, Berlin Heidelberg. DOI 10.1007/s00340-016-6482-0.

[16] B. Wang, B.Y. Gong, M. Wang, B. Weng, and X.P. Zhao, "An Ultrawideband Ultrathin Metamaterial Absorber Based on Circular Split Rings," App. Phys. A., vol. 5, no. 145, 2014.

[17] S. Ghosh, D. Chaurasiya, S. Bhattacharyya, A. Bhattacharya, and K. V. Srivastava. "Dendritic-Metasurface-Based Flexible Broadband Microwave Absorber," IEEE Antennas and Wireless Propagation Letters, vol. 14, pp. 1172-1175, 2015.

[18] J. Tak and J. Choi, "A Wearable metamaterial microwave absorber," IEEE Antennas and Wireless Propagation Letters, vol. 16, pp. 784-787, 2017.

[19] W. Mei, W. Bin, Z. Jing, and Z. Xiaopeng, "Dendritic-Metasurface-Based Flexible Broadband Microwave Absorber," Springer, Berlin Heidelberg, 2017. 